\title{
FIBRE BUNDLES AND MEASURE
}

\author{
BERNARD R. GELBAUM ${ }^{1}$
}

In the study of Banach algebra bundles [2], there arises quite naturally the question of integration of bundle sections if the fibre is a vector space and the base space supports a measure. The possibility of such integration follows from the theorem below. It is published separately because of its independent interest and because of its relevance to the following results in topology.

1. (Doyle-Hocking). Every topological closed $n$-manifold $M^{n}$ is the union of an open $n$-cell and a closed $(n-1)$-dimensional subset disjoint from the cell [1].

2. (BRown-CASSLER). Every closed topological $n$-manifold $M^{n}$ is the continuous image by a map $\phi$ of the closed $n$-cell $I^{n}$ $\equiv[0,1] \times[0,1] \times \cdots \times[0,1]$ (n factors) so that (i) $\phi \mid \partial I^{n}$ is a homeomorphism $\left(\partial I^{n}=I^{n} \bigcap\left(R^{n} \backslash I^{n}\right)^{-}\right.$, (ii) $\phi^{-1} \phi\left(\partial I^{n}\right)=\partial I^{n}$ and (iii) $\operatorname{dim} \phi\left(\partial I^{n}\right) \leqq n-1[1]$.

3. Any (coordinate) bundle $B$ over the $n$-sphere $S^{n}$ is strictly equivalent to a (coordinate) bundle $B^{\prime}$ in normal form. In $B^{\prime}$ the open covering of $S^{n}$ consists of two sets $V_{1}$ and $V_{2}$ each of which is a zone of $S^{n}$ and such that $V_{1} \cap V_{2}$ is a narrow equatorial band. The width of the band can be made arbitrarily small [4].

Result 2 is regarded as complementary to 1 . Results 1 and 3 show that fibre bundles over many manifolds are really fibre bundles over contractible sets united with sets of small dimension or small measure. Such bundles are trivial over the contractible parts of their base spaces. Consequently, each may be exemplified by a coordinate bundle for which the transition functions (coordinate transformations) are constant and equal to the group identity over "most" of the base space.

The theorem of this paper extends the results above. Broadly paraphrased, the theorem says that a fibre bundle over any topological space $X$ endowed with a reasonable topological measure may be exemplified by a coordinate bundle whose transition functions are, on most of $X$, constant and equal to the identity of the group of the bundle. More precisely we have the

THEOREM. Let $\varepsilon$ be a fibre bundle with base space $X$, fibre $F$, group $G$, bundle space $E$ and projection $p: E \rightarrow X$. Assume that on the $\sigma$-ring $\delta$

Received by the editors July 9, 1968.

1 Some of this work was supported by National Science Foundation Grant GP. 5436 to which the author is grateful. 
generated by the compact sets of $X$ there is defined a regular measure $\mu$ and that $X$ has a neighborhood basis $\mathscr{X}=\{N\}$ satisfying $\mu(\partial N)=0$ for all $N \in \Re$, where $\partial N=\bar{N} \cap(X \backslash N)^{-}=$boundary of $N$. Let $K$ be a compact set in $X$ and let $\epsilon>0$. Then there is a coordinate bundle $B^{0}$ in the equivalence class defining $\&$ and a compact set $\tilde{K} \subset K$ such that

1. $\mu(K \backslash \tilde{K})<\epsilon$.

2. If $U^{\prime}, U^{\prime \prime}$ are open sets used in the definition of $B^{0}$ and if $x \in U^{\prime}$ $\cap U^{\prime \prime} \cap \tilde{K}$, then $g_{U^{\prime} U^{\prime \prime}}(x)=e \equiv$ identity of $G$. Here $g_{U^{\prime} U^{\prime \prime}}: U^{\prime} \cap U^{\prime \prime} \rightarrow G$ is the transition function or coordinate transformation corresponding to $U^{\prime}$ and $U^{\prime \prime}$ [4].

REMARKs. (i) If $X$ is a locally compact group and if $\mu$ is Haar measure, then the conditions given above for $X$ and $\mu$ are fulfilled [3].

(ii) If a second coordinate bundle $B^{1}$ in the equivalence class of $\mathcal{E}$ consists, in particular, of an open covering that is a refinement of the open covering used for $B^{0}$ and of maps $\phi_{U}^{1}$ that are restrictions of the corresponding maps $\phi_{U}^{0}$ (see below), then $B^{1}$ also enjoys properties 1 and 2 above.

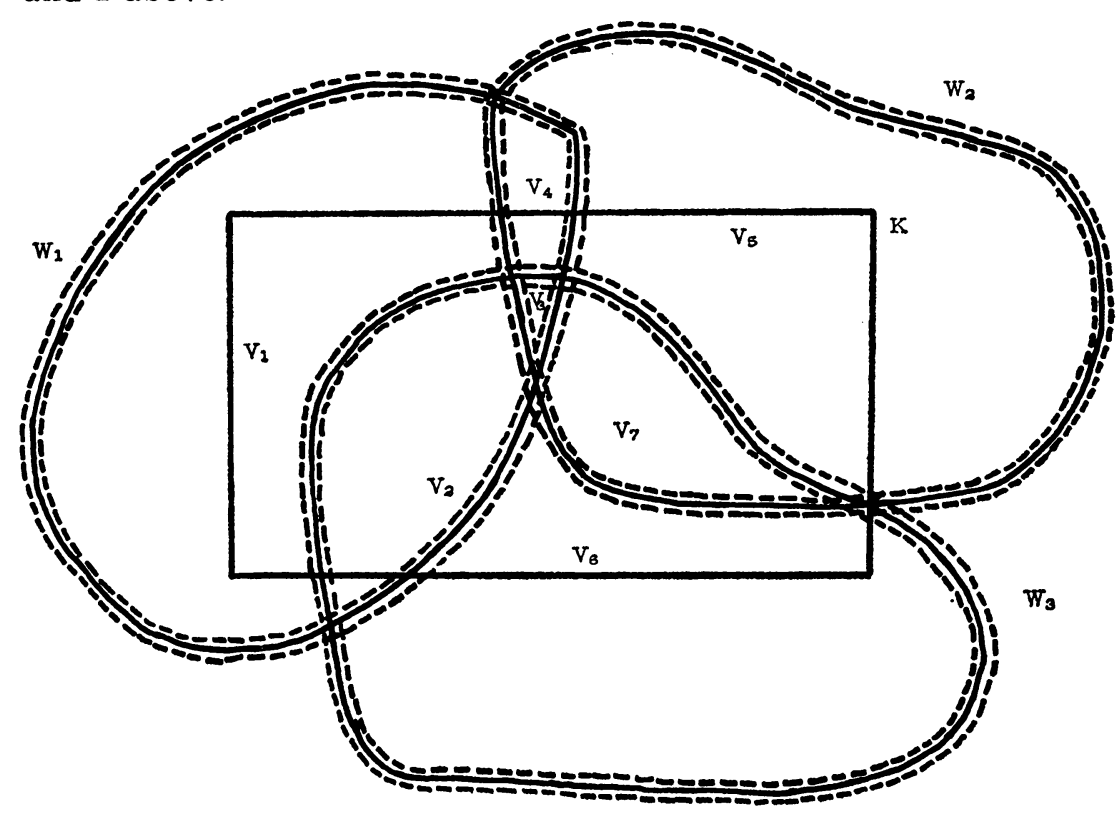

Narrow band defined by dashed line is

$\bigcup_{j} M_{j}$ 
Proof. In what follows, if $U$ is an open set used in the definition of a coordinate bundle then by definition,

$$
\phi_{U}^{-1}: p^{-1}(U) \rightarrow U \times F
$$

is the homeomorphism corresponding to local triviality. If $\mathcal{u}$ is an open covering used in the definition of a coordinate bundle $B^{0}$, and if $\mathcal{V}$ is a refinement of $\mathcal{u}$ let $V \ni V \rightarrow U(V) \in \mathcal{u}$ be a map such that $V \subset U(V)$. Then we define $\phi_{V}^{-1}$ as the restriction to $p^{-1}(V)$ of $\phi_{U(V)}^{-1}$. In this way $V$ and the maps $\phi_{V}^{-1}$ become ingredients of a coordinate bundle $B^{1}$ equivalent to $B^{0}[4]$. The idea of the proof can be followed most readily by use of the diagram provided.

Owing to our hypotheses, we may assume we have a coordinate bundle whose covering $W$ consists of open sets $W$ such that $\mu(\partial W)=0$. We cover $K$ with finitely many sets $W_{1}, W_{2}, \cdots, W_{n}$ of $w$. We then choose a refinement $\mathfrak{T}=\{M\}$ of $W$ so that we may cover $\bigcup_{i=1}^{n}\left(\partial W_{i}\right)$ by a family $\left\{M_{j}\right\}$ of sets of $\mathscr{T}$ and so that

$$
\mu\left(\bigcup_{j} M_{j}\right)<\epsilon
$$

(The regularity of $\mu$ makes this possible.) The set $K \backslash\left(\cup_{j} M_{j}\right) \equiv \tilde{K}$ is compact and $\mu(K \backslash \tilde{K})<\epsilon$.

For each $x \in K \backslash \bigcup_{i=1}^{n} \partial W_{i}$, let $S(x)=\left\{i: x \in W_{i}\right\}$. Then let

$$
V(x)=\left(\bigcap_{i \in S(x)} W_{i}\right) \cap\left(\bigcap_{j \notin S(x)}\left(X \backslash \bar{W}_{j}\right)\right) .
$$

The set $V(x)$ is open. Furthermore, $x \in V(x)$. Otherwise, $x \in \bar{W}_{j}$ for some $j \notin S(x)$. Since $x \notin W_{j}$, we see $x \in \partial W_{j}$, a contradiction. Thus $\bigcup_{x} V(x) \supset K \backslash \bigcup_{i=1}^{n} \partial W_{i}$.

There are only finitely many distinct $V(x)$; let them be $V_{1}, V_{2}$, $\cdots, V_{m}$. They are pairwise disjoint. Indeed let

$$
\begin{aligned}
& V_{p}=\left(\bigcap_{i \in S_{p}} W_{i}\right) \cap\left(\bigcap_{j \notin S_{p}}\left(X \backslash \bar{W}_{j}\right)\right), \\
& V_{q}=\left(\bigcap_{i \in S_{q}} W_{i}\right) \cap\left(\bigcap_{j \notin S_{q}}\left(X \backslash \bar{W}_{j}\right)\right) .
\end{aligned}
$$

If $S_{p}=S_{q}$ then $V_{p}=V_{q}$. If $V_{p} \neq V_{q}$ then $S_{p} \neq S_{q}$, and so there is a $j$ say in $S_{p}$ and not in $S_{q}$. Whence if $x \in V_{q}$, then $x \in X \backslash \bar{W}_{j}$ and so $x \notin W_{j}$, $x \notin V_{p}$. Hence if $V_{p} \neq V_{q}$ then $V_{p} \cap V_{q}=\varnothing$. If a $V_{k}$ meets a $W_{i_{0}}$, then 
$V_{k} \subset W_{i_{0}}$. Indeed, if $V_{k}=\left(\bigcap_{i \in S_{k}} W_{i}\right) \cap\left(\bigcap_{j \notin S_{k}}\left(X \backslash \bar{W}_{j}\right)\right)$ and if $V_{k}$ meets $W_{i_{0}}$, then $i_{0} \in S_{k}$ and thus $V_{k} \subset W_{i_{0}}$.

In summary:

(i) $\bigcup_{k=1}^{m} V_{k} \supset K \backslash \bigcup_{i=1}^{n} \partial W_{i}$.

(ii) $V_{p} \cap V_{q}=\varnothing$ if $p \neq q$.

(iii) Each $V_{p} \subset$ some $W_{i}$ and if $V_{p} \cap W_{i} \neq \varnothing$, then $V_{p} \subset W_{i}$.

Let $\mathcal{U}$ be the family $\{U\}$ consisting of (a) the open sets $\left\{M_{j}\right\}$; (b) for any $M$ meeting $\tilde{K}$, all nonempty sets $M \cap V_{k}$; (c) all nonempty sets $M \backslash \tilde{K}$. Then $\mathfrak{u}$ is an open covering. For if $x \in X$, either $x \in \bigcup_{j} M_{j}$ or $x \in \tilde{K}$, whence $x \in$ some $M$ and $x \in$ some $V_{k}$ whence $x \in$ some $M \cap V_{k}$, or $x \notin \tilde{K}$ and then $x \in$ some $M$ whence $x \in$ some $M \backslash \tilde{K}$. Since each $U \subset$ some $M$, we see $\mathcal{U}$ is a refinement of $M$, hence a refinement of w.

We well-order $W:\left\{W_{\lambda}: 1 \leqq \lambda<\xi\right\}$, where $\xi$ is some initial ordinal. Note that the first $n$ elements of $w$ are the $W_{1}, W_{2}, \cdots, W_{n}$ above. Then for each $U \in \mathcal{U}$ let $\lambda(U)=\min \left\{\lambda: U \subset W_{\lambda}\right\}$. This provides a map $\mathcal{U} \ni U \rightarrow W_{\lambda(U)} \in W$ and thus permits the definition of a coordinate bundle (a) based on $\mathcal{u}$, (b) defined via restrictions of the coordinate maps $\phi_{W}$ given for $W$ and (c) equivalent to the coordinate bundle based on $W$.

Consider an $x \in U^{\prime} \cap U^{\prime \prime} \cap \tilde{K}$ where $U^{\prime}, U^{\prime \prime} \in \mathcal{~}$. Since $x \in \tilde{K}$ we see $x \notin U_{j} M_{j}$. Thus neither $U^{\prime}$ nor $U^{\prime \prime}$ is a set $M_{j}$ nor a set $M_{j} \cap V_{k}$ nor a set $M \backslash \tilde{K}$. Thus we see that, for some $p$ and $q, U^{\prime}=M^{\prime} \cap V_{p}, U^{\prime \prime}$ $=M^{\prime \prime} \cap V_{q}$. Since $x \in U^{\prime} \cap U^{\prime \prime} \neq \varnothing$, we see $V_{p} \cap V_{q} \neq \varnothing$, whence $V_{p}=V_{q} \equiv V_{k}$. We assert that $\lambda\left(U^{\prime}\right)=\lambda\left(U^{\prime \prime}\right)$. Indeed, clearly $\lambda\left(U^{\prime}\right)$, $\lambda\left(U^{\prime \prime}\right) \leqq n$. Furthermore, if $U^{\prime} \subset W_{i}$, then $V_{k}$ meets $W_{i}, V_{k} \subset W_{i}$ (by (iii)) and thus $U^{\prime \prime} \subset W_{i}$. Thus

$$
\left\{\lambda: \lambda \leqq n, U^{\prime} \subset W_{\lambda}\right\}=\left\{\lambda: \lambda \leqq n, U^{\prime \prime} \subset W_{\lambda}\right\},
$$

whence $\lambda\left(U^{\prime}\right)=\lambda\left(U^{\prime \prime}\right)$. Call their common value $r$.

It is now clear that

$$
\phi_{U^{\prime}}^{-1}\left(p^{-1}\left(U^{\prime}\right)\right)=\phi_{W_{r}}^{-1}\left(p^{-1}\left(U^{\prime}\right)\right) \text { and } \phi_{U^{\prime \prime}}^{-1}\left(p^{-1}\left(U^{\prime \prime}\right)\right)=\phi_{W_{r}}^{-1}\left(p^{-1}\left(U^{\prime \prime}\right)\right)
$$

whence $g_{U^{\prime} U^{\prime \prime}}(x)=e$.

CoROLlaRY. If $X \in \mathcal{S}$ and if $\mu(X)<\infty$ then for $\epsilon>0$ there is an open set $W \in \mathcal{S}, \mu(W)<\epsilon$ and, for any refinement of the coordinate bundle indicated in the theorem, $g_{U^{\prime} U^{\prime \prime}}(x)=$ identity if $x \in U^{\prime} \cap U^{\prime \prime} \cap(X \backslash W)$.

The above theorem permits the development of an integration theory in appropriate contexts. We propose to discuss this theory and its application to "group algebra bundles" in a separate paper. 


\section{BIBLIOGRAPHY}

1. Morton Brown, "A mapping theorem for untriangulated manifolds" in Topology of 3-manifolds, and related topics, Prentice-Hall, Englewood Cliffs, N. J., 1962, pp. 92-94.

2. B. R. Gelbaum, Banach algebra bundles, Pacific J. Math. (to appear).

3. C. S. Herz, The spectral theory of bounded functions, Trans. Amer. Math. Soc. 94 (1960), 181-232.

4. N. Steenrod, The topology of fibre bundles, Princeton Univ. Press, Princeton, N. J., 1960.

UNIVERSity of CALIFornia, IRVINE 